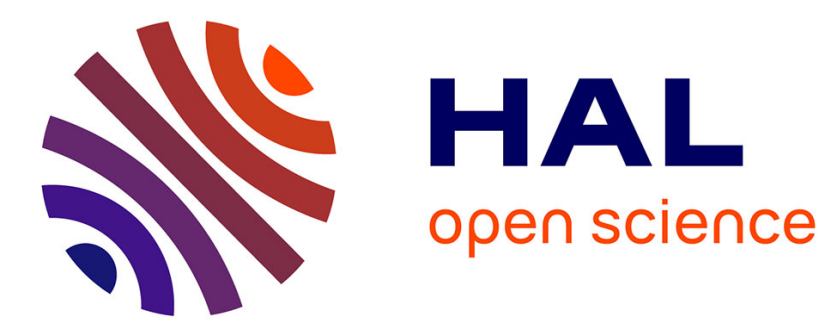

\title{
The dialogical dynamics of adaptive paraconsistency.
}

\author{
Shahid Rahman, Jean-Paul Bendegem
}

\section{To cite this version:}

Shahid Rahman, Jean-Paul Bendegem. The dialogical dynamics of adaptive paraconsistency.. A. Carnielli, M. Coniglio, I. M. Loffredo D'Ottaviano (eds.). Paraconsistency, the logical way to the inconsistent, Marcel Dekker, pp.295-322., 2002. halshs-01228976

\section{HAL Id: halshs-01228976 https://shs.hal.science/halshs-01228976}

Submitted on 15 Nov 2015

HAL is a multi-disciplinary open access archive for the deposit and dissemination of scientific research documents, whether they are published or not. The documents may come from teaching and research institutions in France or abroad, or from public or private research centers.
L'archive ouverte pluridisciplinaire HAL, est destinée au dépôt et à la diffusion de documents scientifiques de niveau recherche, publiés ou non, émanant des établissements d'enseignement et de recherche français ou étrangers, des laboratoires publics ou privés. 


\title{
The Dialogical Dynamics of Adaptive Paraconsistency $*$
}

\author{
SHAHID RAHMAN \\ JEAN PAUL VAN BENDEGEM
}

University of Lille 3, France rahman@,univ-lille3.fr

University of Brussels, Belgium jpvbende@,vub.ac.be

\begin{abstract}
Abs tract
The dialogical approach to paraconsistency as developed by Rahman and Carnielli ([1]), Rahman and Roetti ([2]) and Rahman ([3], [4] and [5]) suggests a way of studying the dynamic process of arguing with inconsistencies.

In his paper on Paraconsistency and Dialogue Logic ([6]) Van Bendegem suggests that an adaptive version of paraconsistency is the natural way of capturing the inherent dynamics of dialogues. The aim of this paper is to develop a formulation of dialogical paraconsistent logic in the spirit of an adaptive approach and which explores the possibility of eliminating inconsistencies by means of logical preference strategies.
\end{abstract}

\section{INTRODUCTION}

The dialogical approach to paraconsistency as developed by Rahman and Carnielli ([1]), Rahman and Roetti ([2]) and Rahman ([3]), ([4]) and ([5]) suggests a way of studying the dynamic process of arguing with inconsistencies.

In his paper on Paraconsistency and Dialogue Logic ([6]) Van Bendegem, who supports the dialogical approach to paraconsistency, argues however that Rahman-Carnielli's formulation defeats the aim of a general dynamic structure of dialogical paraconsistent logics because their formulation uses a paraconsistency rule which applies not only to arguments containing inconsistent formulae but also to arguments where no inconsistent formulae occur. Van Bendegem suggests that an adaptive version of paraconsistency is the natural way of capturing the inherent dynamics of dialogues. In fact, Diderik Batens proposed in his paper A survey of inconsistency-adaptive logics ([7]) that the study of adaptive logic should be carried out in the context of argumentation.

The aim of this paper is to develop a formulation of dialogical paraconsistent logic in the spirit of an adaptive approach and which explores the possibility of eliminating inconsistencies by means of logical preference strategies.

\section{THE DIALOGICAL LITERAL APPROACH TO PARACONSISTENCY AND ITS PROBLEMS}

\subsection{Lite ral Paraconsistency}

One way to formulate paraconsistent logic within the dialogical approach as developed in Rahman and Carnielli ([1]), Rahman and Roetti ([2]) and Rahman ([4]) can be achieved in the following way. Assume that to the structural rules of the standard dialogical logic- - -we add the following:

- Negative Literal Rule:

The Proponent is allowed to attack the negation of an atomic (propositional) statement (the so called negative literal) if and only if the Opponent has already attacked the same statement before.

This structural rule can be considered in analogy to the formal rule for positive literals. The idea behind this rule is the following: An inconsistency of the Opponent may be tolerated by using a type of charity principle. The inconsistency might involve different semantic contexts in which, say, $a$ and $\neg a$ have been asserted. Now, if the Opponent attacks $\neg a$ with $a$ he concedes thereby that there is some common context between $\neg a$ and $a$ which makes an attack on $\neg a$ possible. This allows the Proponent to attack the corresponding negation of the Opponent.

In Rahman and Carnielli ([1]) the logics produced by this rule were called Literal Dialogues, or shorter: L-D. In order to distinguish between the intuitionistic and the classical version Rahman and Carnielli wrote L-D ${ }^{\mathrm{i}}$ (for 
the intuitionistic version) and $\mathrm{L}-\mathrm{D}^{\mathrm{c}}$ (for the classical version). To be precise we should call these logical systems literal dialogues with classical structural rules and literal dialogues with intuitionistic structural rules respectively.

In L-D the (from a paraconsistent point of view) dangerous formulae $(a \wedge \neg a) \rightarrow b, a \rightarrow(\neg a \rightarrow b)$ and $(a \rightarrow b) \rightarrow((a \rightarrow \neg b) \rightarrow \neg a)$ are not valid. Let us see the corresponding literal dialogues in L-D ${ }^{\mathrm{c}}$ for the first one:

\section{Example 1}

\begin{tabular}{ll|lll}
$\mathrm{O}$ & \multicolumn{3}{|c}{$\mathrm{P}$} \\
\hline & & & $(a \wedge \neg a) \rightarrow b$ & (0) \\
(1) $\quad a \wedge \neg a$ & 0 & & & (2) \\
(3) $a$ & & 1 & ? & (4) \\
(5) $\neg a$ & & 1 & &
\end{tabular}

The Proponent loses because he is not allowed to attack the move (5) (see negative literal rule). In other words the Opponent may have contradicted himself, but the semantic context of the negative literal is not available to the Proponent until the Opponent starts an attack on the same negative literal - an attack which in this case will not take place.

All classically valid formulae without negation are also valid in $\mathrm{L}-\mathrm{D}^{\mathrm{c}}$. All intuitionistically valid formulae without negation are also valid in $L-D^{i}$. As in da Costa's system $C_{1}$ neither of the following is valid in $L-D^{c}$ :

$$
\begin{aligned}
& (a \wedge \neg a) \rightarrow b \\
& (a \wedge \neg a) \rightarrow \neg b \\
& \neg(a \wedge \neg a) \\
& a \rightarrow \neg \neg a \\
& (a \rightarrow b) \rightarrow((a \rightarrow \neg b) \rightarrow \neg a) \\
& ((a \rightarrow b) \wedge(a \rightarrow \neg b)) \rightarrow \neg a \\
& ((\neg a \rightarrow b) \wedge(\neg a \rightarrow \neg b)) \rightarrow a \\
& \neg a \rightarrow(a \rightarrow b) \\
& \neg a \rightarrow(a \rightarrow \neg b) \\
& a \rightarrow(\neg a \rightarrow b) \\
& a \rightarrow(\neg a \rightarrow \neg b) \\
& ((a \rightarrow \neg a) \wedge(\neg a \rightarrow a)) \rightarrow b
\end{aligned}
$$

$$
\begin{aligned}
& (a \rightarrow(b \vee c)) \rightarrow((a \wedge \neg b) \rightarrow c) \\
& ((a \rightarrow \neg a) \wedge(\neg a \rightarrow a)) \rightarrow \neg b \\
& ((a \wedge b) \rightarrow c) \rightarrow((a \wedge \neg c) \rightarrow \neg b) \\
& ((a \vee b) \wedge \neg a)) \rightarrow b \\
& a \vee b) \rightarrow(\neg a \rightarrow b) \\
& (a \rightarrow b) \rightarrow(\neg b \rightarrow \neg a) \\
& (a \rightarrow b) \vee(\neg a \rightarrow b) \\
& (\neg a \vee \neg b) \rightarrow \neg(a \wedge b) \\
& (\neg a \wedge \neg b) \rightarrow \neg(a \vee b) \\
& (\neg a \vee b) \rightarrow(a \rightarrow b) \\
& (a \rightarrow b) \rightarrow \neg(a \wedge \neg b) \\
& a \rightarrow((\neg a \vee b) \rightarrow b)
\end{aligned}
$$

In $\mathrm{L}-\mathrm{D}^{\mathrm{i}}$ all the intuitionistically non-valid formulae have to be added to the list, for example:

$$
\begin{array}{ll}
\neg \neg A \rightarrow A & A \vee(A \rightarrow B) \\
A \vee \neg A & A \vee((A \vee B) \rightarrow B) \\
((A \rightarrow B) \rightarrow A) \rightarrow A & \neg(A \rightarrow B) \rightarrow A
\end{array}
$$

Validity is defined in dialogical logic via winning strategies for the Proponent. A systematic description of the winning strategies for these logics can be obtained from appropriate tableau systems. ${ }^{[\text {[i] }}$-The extension of literal dialogues for propositional logic to first-order quantifiers is straightforward: We only have to extend the structural negative literal rule to elementary statements of first-order logic.Now we shall consider the problems.

\subsection{Problems of the Literal Approach}

The large number of non-valid formulae listed above suggests that the literal approach presented is too restrictive. There are many formulae in this list we would like to have as valid. A further problem is what to do with inconsistencies between complex propositions. One could argue that contradictions which cannot be 
carried on at the literal level should be released of paraconsistency restrictions - this was defended in Rahman ([3], [4] and [5]) where a distinction between internal or de re and external or de dicto negation was introduced. One can also follow another complementary strategy and introduce a device combining two types of restrictions, one applying to the literal case and the other to the complex case. Thus, it looks like more has to be done than just introducing a structural rule restricting the use of negations. One first idea is to introduce an adaptive extension of the literal negative rule making use of the dynamics of argumentation. That is, one argues consistently as long as possible and when necessary adapts to the specific inconsistencies that occur. In other words, the consistency of all sentences is presupposed unless and until proven otherwise (cf. Batens [7], 1). This fits nicely with the problem of complex inconsistencies: If one analyses the complex inconsistency until the literal level has been reached and no inconsistency occurs, then one continues using a non-paraconsistent framework. But as remarked by Max Urchs the adaptive logic of Batens has another deep and appealing contribution to paraconsistency: What is really dangerous is not inconsistency as such, but contradictions (the latter are also called explosive inconsistencies) ([8]). Overlooking such a distinction leads to a uniform treatment of some types of formulae which should be kept apart - e.g. the inconsistency which can occur in a dialogue for disjunctive syllogism or de Morgan should be distinguished from the inconsistency produced by ex falso sequitur quodlibet. The idea is that if you have an inconsistency in the premises you can either isolate it and continue with the rest or take that part of the inconsistency which is necessary for the proof - this strategy has been called by Batens the logical preferential mechanism - in contrast to the non-logical preferential mechanisms of standard non-monotonic logics. Now what distinguishes inconsistencies from contradictions? Our proposal is the following: While contradictions (i.e. explosive inconsistencies) in the concessions of an argument make at least one defensive or aggressive move redundant, non-explosive inconsistencies do not. That is why contradictions lead in this approach to the paraconsistent non-validity of those formulae in which they occur. Moreover, some parts of the (non-explosive) inconsistencies can be used for the defence of the argument (the other parts can be dismissed). Thus, an appropriate concept of redundancy should be introduced here. This is the line we are going to follow in this paper.

\section{THE DIALOGICAL WAY TO ADAPTIVE PARACONSISTENCY AT THE GAME LEVEL}

As mentioned above, we introduce here a redundancy concept which can be applied in order to distinguish between non-explosive inconsistencies and contradictions. Clearly, this procedure should be understood adaptively. That is, if no inconsistencies are present, use the standard definition of dialogical validity (see the appendix). If inconsistencies appear, restrict the definition of validity by means of the redundancy principle.

\subsection{Formulae Which are Paracons is tently Free of Redundancies: Dialogical Adaptivity}

We will present here one concept of redundancy which is a variation of some ideas on dialogical relevance logic developed in Rahman and Rückert [9]. In fact other versions based on relevance considerations introduced in the above-mentioned paper could also be applied. We have chosen this version because it seems more inherent to the paraconsistent point of view in the sense that it stresses the point of having inconsistency without triviality.

The idea behind our dialogical approach to adaptivity is that in the case an inconsistency occurs the Proponent can try one of the following procedures:

i) He may try to win by isolating the inconsistency. That is, he will just ignore it and continue with the rest. If he can answer all the attacks of the Opponent anyway the inconsistency was redundant and he is through.

ii) He may try to win using only one part of the inconsistency and continue with the rest just as in $\mathrm{i}$.

iii) He may try to win using the inconsistency and ignoring the rest of the moves of the Opponent.

We will say that the inconsistency is explosive and the thesis not paraconsistently valid iff the third case is the only successfully proceeding available. Shorter, we call explosive such inconsistencies which make a defensive move redundant.

Now, what happens in the case of a negation occurring in the then-part of a conditional, as in $(a \wedge \neg a) \rightarrow \neg r$ ? Well, if nothing is added we obtain a minimal logic; if we want more than minimality then here again various 
options are available. We will discuss an option which tolerates inconsistencies, does not lead to triviality but renders the principle of non-contradiction as valid. The idea is that a non-explosive inconsistency should not make any defensive move redundant, that is, the Proponent has to use all his defensive moves. (Counterattacks resulting from an attack on negations are considered to be defensive moves.) Suppose the Proponent states the thesis $\neg(a \wedge \neg a)$. The Opponent will attack with move $(\mu)(a \wedge \neg a)$. The Proponent should now use this move. Now, this leads to an awkward formulation where two types of structural rules have to be distinguished: one applying to attacks and the other to counterattacks. A more general approach results from the following considerations: Suppose, on one hand, that the Proponent notices that he can win without using (or with only one part of) the inconsistency which appears in a conjunction stated by the Opponent, then he should be allowed to attack only one part of this conjunction. Suppose, on the other hand, that the Proponent notices that he can win without using (or with only one part of) the inconsistency which appears in a disjunction stated by himself, then he should be allowed to defend only one part of this disjunction

Thus, if an inconsistency in the concessions occurs, the Proponent must attack each formula at least once, use all the atomic formulae of the Opponent and defend himself against all attacks. It is time to be a little more precise:

DEFINITION 1: O-Variants: Any possible development of a dialogue as determined by the choice of the Opponent constitutes a variant. Thus, the developments of a dialogue as determined by the choice between attacks ?L and ?R, or ?R after L? on a conjunction, the different possible defences of a disjunction (left or right side) and the choice between defence and counterattack while reacting to an attack on a conditional are said to be different $O$-variants.

DEFINITION 2: O-Inconsistency: It is said that an O-inconsistency occurs iff

1. The Opponent stated $(v) A, \neg(\mu) A$, where $v$ and $\mu$ signalise the numbers of the respective moves and $A$ is a formula.

2. The Opponent stated a negative refutable formula (like $\neg(A \vee \neg A) ; \neg(A \rightarrow A)$ )

Notice that here we make paraconsistency for complex formulae possible. Clearly, a more restrictive definition applying to literals is possible.

If we want to put the idea of adaptivity into practice we need to precise the notion of used

1. An atomic O-formula has been used iff the Proponent states this formula in order to state an aggressive (=attack) or a defensive move.

2. A complex formula $A$ has been used iff all the aggressive and defensive moves related to $A$ have been stated according to the conditions stated by definition 3 (below).

DEFINITION 3: A formula is said to be free of paraconsistent redundancies iff the Proponent wins under the standard structural rules and he can win under the following conditions:

1. He can attack every O-formula at least once (e.g. he can win with only one of the possible attacks on a conjunction) in any of the possible $\mathrm{O}$-variants (not necessarily in the same $\mathrm{O}$-variant).

2. He can defend himself at least once against all attacks (e.g. he can win defending only one part of the disjunction) in any of the possible $\mathrm{O}$-variants (not necessarily in the same O-variant)

3. He can use at least one occurrence of any atomic O-formula in any of the possible O-variants (not necessarily in the same O-variant).

DEFINITION 4: A formula is said to contain paraconsistent redundancies iff the Proponent cannot win without dismissing at least one of the conditions stated before.

We will tag all attacked O-formulae and all atomic O-formulae used. We do not need to tag the defences because they can be recognised by the closed rounds - see appendix.

Now the definition of formulae which are valid by adaptation:

DEFINITION 5: A formula in the dialogical proof of which an inconsistency occurs is valid by adaptation if this formula is valid by the standard definition of validity and is free of paraconsistent redundancies. Inconsistencies occurring in the dialogical proof of formulae not paraconsistently free of redundancies are called explosive inconsistencies or contradictions.

Let us study some examples. In example 1 of chapter 1.1 (played in standard dialogical logic) it is clear that the inconsistency occurring in the concessions (O-formulae) is explosive: The Proponent wins according to the standard definition of validity, but there is no variant where the Proponent can win by stating the (redundant) 
defence $b$. In the next two examples the inconsistencies do not collapse into contradictions.

Example 2

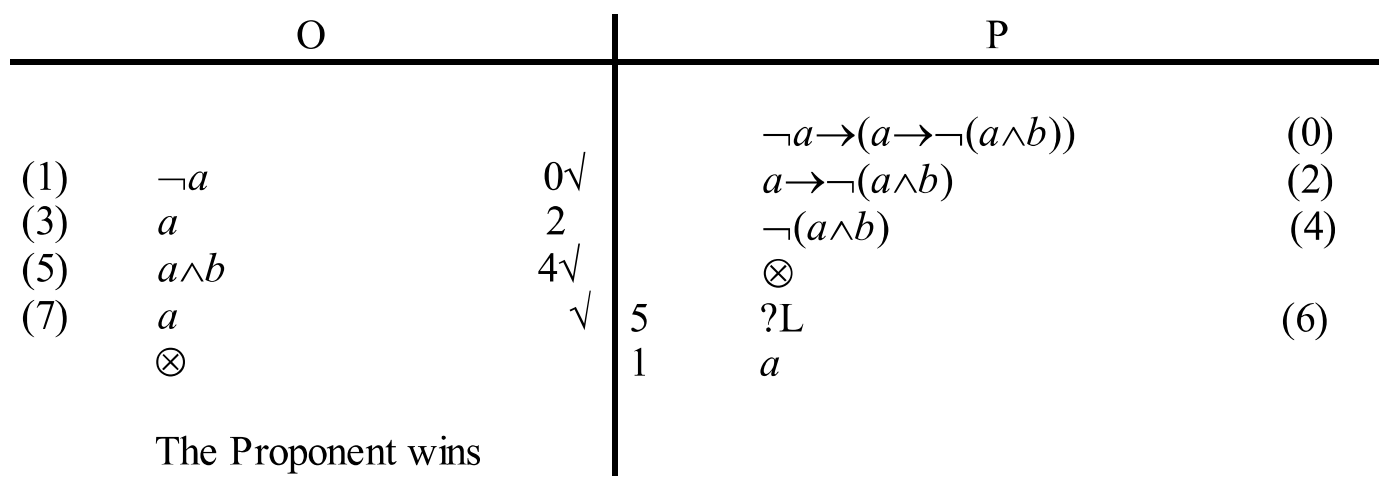

Because of the inconsistency occurring in 1, 3 the Proponent plays adaptively. In doing so, in a first stage he ignores the inconsistency. Then he uses move 7 (or move 3) to attack $\neg a$ and wins. Moreover, since there is no other variant to be considered the formula is valid.

It should not be difficult for the reader to check that neither $\neg a \rightarrow(a \rightarrow b)$ nor $(\neg a \wedge a) \rightarrow b$ hold and that $\neg(\neg a \wedge a), \neg a \rightarrow(a \rightarrow \neg a),(\neg a \wedge a) \rightarrow a$ and $(a \rightarrow \neg \neg a)$ hold.

The next example will lead us to determine how to check whether a given formula is redundant. In this example two variants have to be considered. In order to keep track of the possible redundancies at the game level we will introduce subdialogues (the tableau system due to be introduced later will include all the variants, but at the strategy level).- ${ }^{[\mathrm{iv}]}$-The idea is quite simple. Suppose that at a given stage of the dialogue, the Opponent, who stated an inconsistency realises that he loses by the standard rules but he realises too that one defensive move has been dismissed. He then can ask something like: Well, I will lose in this way, but what about, say, the defensive move $b$ ? (shorter ?b) You did not show me that I can win using that. In this case the Proponent will open a subdialogue where he will have to show that he can win with the move challenged. Now, it can be the case that the Proponent says: I can win using $b$, but I will use it only if in your variant (i.e., the O-variant) you choose, say, the right part - notice that this is a consequence of condition 3 of definition 3 which states that the Proponent can use one of the defensive answers in only one of the $\mathrm{O}$-variants. This can be formulated with a new structural rule:

R5 (subdialogues): The Opponent may at a given stage $\mu$ of the dialogue ask for some unused formula which has been dismissed so far and which should be used according to definition 3 . The Proponent will have to defend this challenge by asking the Opponent to develop all of his O-variants by means of subdialogues. Those formula of the initial dialogue which define the O-variant can be used in the subdialogue.

Example 3

\begin{tabular}{lll|lll}
\multicolumn{2}{c|}{$\mathrm{O}$} & \multicolumn{3}{|c}{$\mathrm{P}$} \\
\hline (1) & $(a \vee b) \wedge \neg a$ & $0 \sqrt{ }$ & & $(0)$ & $((a \vee b) \wedge \neg a)) \rightarrow b$ \\
$(3)$ & $a \vee b$ & $\sqrt{ }$ & & & $(2)$ \\
$(5)$ & $a$ & $\sqrt{ }$ & 1 & $? \mathrm{~L}$ & $(4)$ \\
$(7)$ & $\neg a$ & $\sqrt{ }$ & 3 & $?$ & $(6)$ \\
& & & & $? \mathrm{R}$ & $(8)$
\end{tabular}

At this stage, where the Opponent is going to lose he realises that round 1 is still open. $O$ then asks for a response to 1 and $\mathrm{P}$ will ask $\mathrm{O}$ to open a subdialogue for the right-side variant of the defence to the attack 4 . In this subdialogue $\mathrm{P}$ will show that $b$ is not redundant because if the Opponent chooses $b$ at move 5 he will answer the attack of move 1 and win. Thus the dialogue now looks as follows:

\begin{tabular}{lll|lll}
\multicolumn{2}{c|}{$\mathrm{O}$} & \multicolumn{3}{c}{$\mathrm{P}$} \\
\hline & & & $((a \vee b) \wedge \neg a)) \rightarrow b$ \\
(1) & $(a \vee b) \wedge \neg a$ & $0 \sqrt{ }$ & $(0)$ & & \\
(3) & $a \vee b$ & $\sqrt{ }$ & & $b$ & (12) \\
(5) & $a$ & $\sqrt{ }$ & 1 & $? \mathrm{~L}$ & (4) \\
(7) & $\neg a$ & $\sqrt{ }$ & 3 & $?$
\end{tabular}




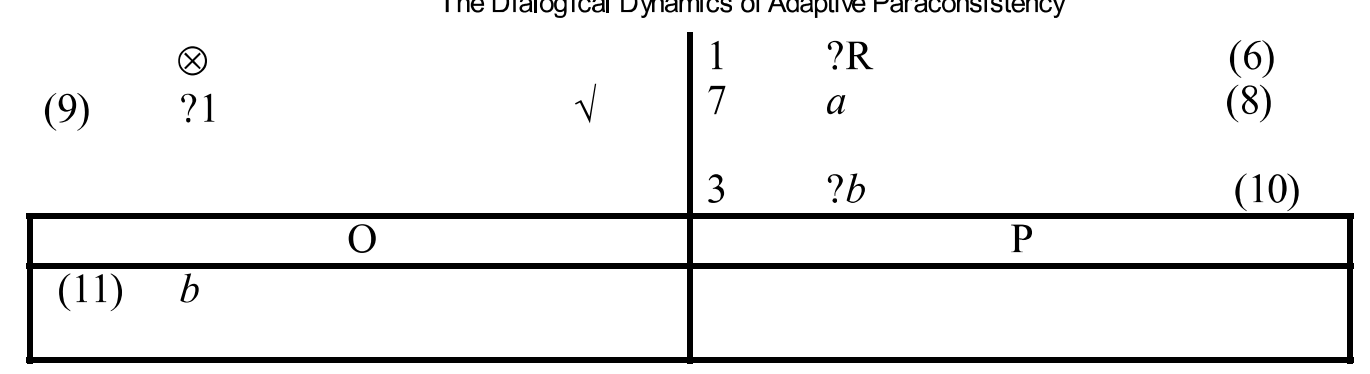

At move 9 the Opponent asks for the round opened at move 1. The Proponent counterattacks by asking for the $\mathrm{O}$-variant $b$ in the defence of the disjunction. After this counterattack the Proponent uses $b$ to close the opened round. Actually we could rewrite all the variant again but we dispense with doing so by allowing jumping from the subdialogue to the initial dialogue.

Notice that a clever Proponent will always try to play classically because the adaptive rules restrict his own moves and not the moves of the Opponent. Thus, the Opponent will try to state an inconsistency as fast as he can in order to play paraconsistently. For example, in the dialogue for $((\neg a \vee b) \wedge a)) \rightarrow a$ the Proponent will not try to produce the $\mathrm{O}$-inconsistency $\neg a, a$. He will instead attack the right part of the conjunction obtaining $a$ and then he will immediately close the first round using precisely this $a$ and win.

\subsection{Quantifiers and Ontological Adaptivity}

The quantifiers do not in this approach present any special problems in relation to inconsistencies. But there are some interesting considerations about the ontological status of inconsistencies. Actually there are two main interpretations of paraconsistency possible. The one, called the compelling interpretation, stresses that paraconsistent theories are ontologically committed to inconsistent objects. The other, called the permissive interpretation, does not assume this ontological commitment of paraconsistent theories. Da Costa formulates this latter approach in the following way:

I suggest to address the inconsistency issue differently. We may well explore the rich representational devices allowed by the use of paraconsistency in inconsistent domains, but withholding any claim to the effect that there are 'inconsistent objects' in reality. (Da Costa [14], $33)$.

This allows for the accommodation of inconsistency by acknowledging that it is not a permanent feature of reality to which theories must correspond, but is rather a temporary aspect of such theories [...]. In this view, to accept a theory is to be committed, not to believing it to be true per se, but to holding it as if it were true, for the purposes of further elaboration, development and investigation. (Da Costa, Bueno and French [15], 616-617).

In Rahman ([4] and [5]) a logic was developed in which the temporary acceptance of inconsistencies will be abandoned as soon as the singular terms occurring in the elementary propositions producing these inconsistencies are ontologically committed. In other words, the Proponent concedes the Opponent's inconsistency $\mathbf{A}_{\tau} \wedge \neg \mathbf{A}_{\tau}$ iff the constant $\tau$ carries no ontological commitment. Actually we are not only differentiating between explosive and non-explosive inconsistencies: we propose here to distinguish between inconsistencies which are ontologically committing and those which are not. This distinction can be considered in the context of $\mathrm{Da}$ Costa's epistemology of quasi truths. The idea is the following. It could well be that, say, $B_{\tau}$ has somehow been verified in reality and thus we commit ontologically with it and reject $\neg B_{\tau}$ But it could also happen that there is no verification either of $A_{\tau}$ or of $\neg A_{\tau}$ and according to our theory it seems we should concede $A_{\tau}$ and $\neg A_{\tau}$ In this case, though we will concede it, we will not take this concession as being ontologically committed.

The introduction of ontologically committing quantifiers can be achieved through the following definition and a new structural rule:

DEFINITION 6: A constant $\tau$ is said to be introduced with ontological commitment by $\mathrm{X}$ if

(1) $\mathrm{X}$ states a formula $A[\tau / \mathrm{x}]$ to defend $\vee_{x} A$ or

(2) $\mathrm{X}$ attacks a formula $\wedge_{x} A$ with ? $v / \tau$, and $\tau$ has not been used in the same way before. 
R6 (formal introduction of constants): P may not introduce constants with ontological commitment: any such constant must first be introduced by $\mathrm{O}$ according to definition 6. (cf. [16])

The intuitive idea behind the ontologically committing quantifiers should be clear: The Proponent is allowed to use a constant for a defence (of an existential quantifier) or an attack (on a universal quantifier) iff the Opponent has already conceded that this constant has ontological commitment by an attack (on a universal quantifier) or by a defence (of an existential quantifier).

This logic also contains the quantifiers $\exists$ and $\forall$ for which neither definition 6 nor R6 hold. Since the quantifiers of $\exists$ and $\forall$ are ruled by the standard and structural rules it looks like these quantifiers work as in the standard logic. Actually they are very different: here they work as quantifiers without any ontological commitment at all. In the standard logic the ontological commitment is presupposed by the use of these quantifiers. Here this is not the case.

Our adaptive rule for quantified adaptive paraconsistent logic is the following:

R7 (ontological adaptivity): If an O-inconsistency occurs and its constants were not introduced according to definition 6 and R6, replace these quantifiers with $\exists$ and $\forall$ and run an adaptive dialogue as described in the definitions 1 to 5 . Proceed classically otherwise.

Example 4

\begin{tabular}{|c|c|c|c|c|}
\hline \multirow[b]{4}{*}{ (1) } & \multirow[b]{4}{*}{$\wedge x((A x \vee B x) \wedge \neg A x))$} & \multirow[b]{4}{*}{$0 \sqrt{ }$} & \multicolumn{2}{|c|}{$\mathrm{P}$} \\
\hline & & & \multicolumn{2}{|c|}{$(\wedge x((A x \vee B x) \wedge \neg A x))) \rightarrow$} \\
\hline & & & $\vee x(\neg \neg B x \vee C \mathrm{x})$ & \\
\hline & & & $\vee x(\neg \neg B x \vee C \mathrm{x})$ & (2) \\
\hline (3) & $?$ & $2 \sqrt{ }$ & & \\
\hline (5) & $(A \tau \vee B \tau) \wedge \neg A \tau$ & & $? \tau$ & (4) \\
\hline (7) & $A \tau \vee B \tau$ & & $? \mathrm{~L}$ & (6) \\
\hline (9) & $A \tau$ & & 7 & (8) \\
\hline (11) & $\neg A \tau$ & & ?R & (10) \\
\hline
\end{tabular}

At moves 9 and 11 an O-inconsistency occurs. The Proponent will concede the inconsistency and thus, run an adaptive dialogue, but he will not take it as ontologically committing:

\begin{tabular}{|c|c|c|c|c|c|}
\hline \multicolumn{3}{|c|}{$\mathrm{O}$} & \multicolumn{3}{|c|}{$P$} \\
\hline $\begin{array}{l}(1) \\
(3) \\
(5) \\
(7) \\
(9) \\
(11) \\
(13)\end{array}$ & $\begin{array}{l}\forall x((A x \vee B x) \wedge \neg A x)) \\
? \\
(A \tau \vee B \tau) \wedge \neg A \tau \\
A \tau \vee B \tau \\
A \tau \\
\neg A \tau \\
\otimes \\
? 3\end{array}$ & $\begin{array}{l}0 \sqrt{ } \\
2 \sqrt{ }\end{array}$ & $\begin{array}{l}1 \\
5 \\
7 \\
5 \\
7\end{array}$ & $\begin{array}{l}(\forall x((A x \vee B x) \wedge \neg A x))) \rightarrow \exists x \\
(\neg \neg B x \vee C \mathrm{x}) \\
\exists x(\neg \neg B x \vee C \mathrm{x}) \\
\neg \neg B \tau \vee C \tau \\
? \tau \\
? \mathrm{~L} \\
? \\
? \mathrm{R} \\
A \tau \\
? B \tau\end{array}$ & $\begin{array}{r}(0) \\
(2) \\
(16) \\
(4) \\
(6) \\
(8) \\
(10) \\
(12) \\
\\
(14)\end{array}$ \\
\hline & $\mathrm{O}$ & & & $\mathrm{P}$ & \\
\hline $\begin{array}{l}\text { (15) } \\
17) \\
\text { (19) } \\
\text { The }\end{array}$ & $\begin{array}{l}B \tau \\
? \\
\neg B \tau \\
\otimes \\
\text { ponent wins }\end{array}$ & $\begin{array}{r}\sqrt{ } \\
16 \sqrt{ } \\
18 \sqrt{ }\end{array}$ & 19 & $\begin{array}{l}\neg B \tau \\
\otimes \\
B \tau\end{array}$ & $\begin{array}{l}\text { (18) } \\
(20)\end{array}$ \\
\hline
\end{tabular}

Now it could well be that we do not want to replace every quantifier automatically. It could be the case that some formulae in which these quantifiers occur are known to have an empirical verification and thus should be 
read as ontologically committing. In fact, the different quantifiers allow us to think about different possible combinations between types of quantifiers. We could, as already mentioned, study the possibility that not all of the quantifiers lose their ontological commitment. This would allow one part to have ontological commitment and the other not. In this case we could, for example, proceed with an anti Meinongian point of view and give priority to the existent. That is, we could understand that if one part of the inconsistency carries ontological commitment the inconsistency cannot hold anymore because this part will be considered to be verified and we should proceed classically. This can be made explicit by copying the quantifiers with ontological commitment in the P-column at the start of the dialogue:

\section{Example 5}

In this example we will not go into the process of replacing. We will instead replace at the start what has not been conceded as ontologically committing:

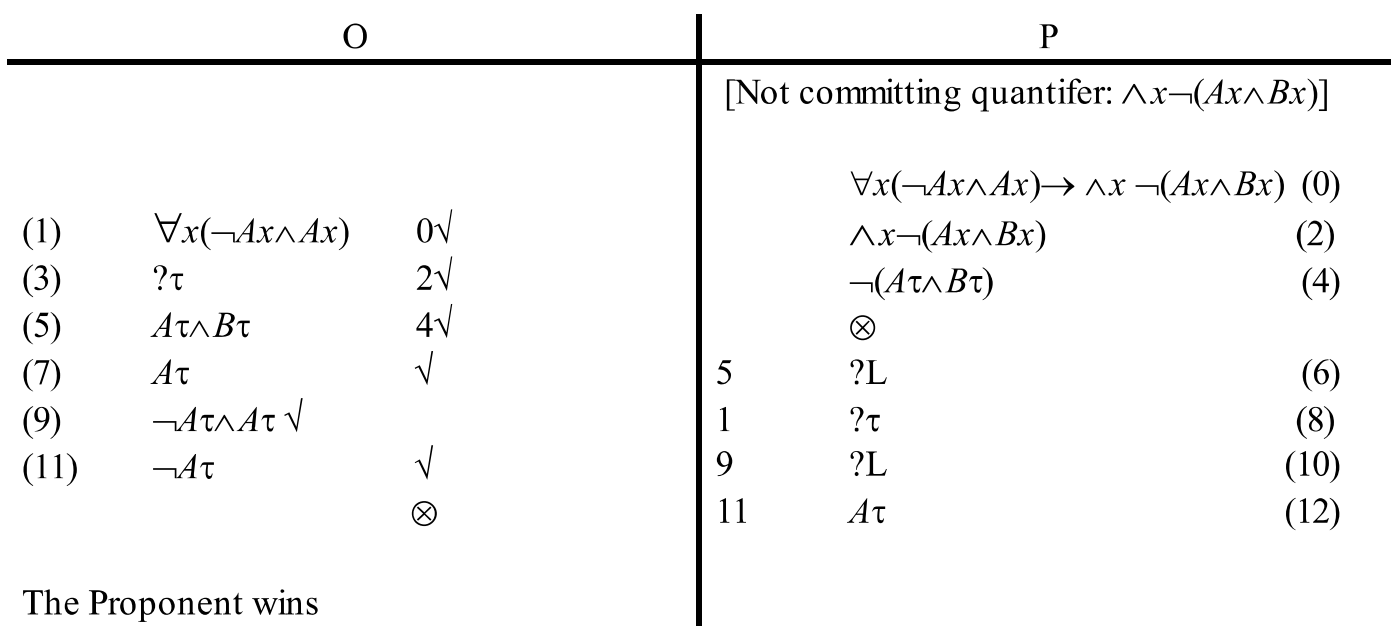

Another possibility due to be explored is to introduce many quantifiers with different grades of ontological commitment or verification. Furthermore it could be that we have a hierarchy of quantifiers corresponding to grades of confirmation. We could in this case require that if a constant of level $v$ has been introduced with the help of quantifiers of level $\mu<\nu$ then this constant should be considered as having ontological commitment in $\mu$. More precisely, think of the first pair of quantifiers as having the upper index 0 and add new pairs of quantifiers with higher indices, as many as we need to express every type of (verified) reality (or fiction) that could possibly appear. The extended set of quantifiers requires a new notion of introduction.

- A constant $\tau$ is said to be introduced with ontological commitment as belonging to the type $\mathrm{i}$ iff it is used to attack a universal quantifier of type $i$ or to defend an existential quantifier of type $i$ and has not been used in the same way before.

- For each type of quantification the following rule holds: Constants may only be introduced by O.

Now the hierarchy:

- P may introduce a constant $\tau$ with ontological commitment on a level $\mu$ iff $\mathrm{O}$ has introduced $\tau$ in the same way on some level $\nu$ with $\nu<\mu$ before.

We leave two examples as an exercise for the reader:

1. $\quad\left(\vee^{1} \mathbf{A}_{x} \vee \vee_{x}{ }^{2} \mathbf{A}_{x}\right) \rightarrow \exists_{x} \mathbf{A}_{x}$

2. $\quad\left(\vee^{1}{ }_{x} \mathbf{A}_{x} \wedge \wedge_{x}{ }^{2}\left(\mathbf{A}_{x} \rightarrow \mathbf{B}_{x}\right)\right) \rightarrow \vee^{1}{ }_{x} \mathbf{B}_{x}$

Thus, in our paraconsistent context we could think of applying the following rule:

- if an $\mathrm{O}$-inconsistency occurs at level $\mu$, check first whether the corresponding constants have been introduced at level $\nu<\mu$. 
If constants have been introduced in $v$ two procedures are possible:

1. stay at level $\mu$, ignore this, and use an adaptive paraconsistency,

2. stay at level $\mu$, proceed classically for the inconsistency with constants introduced at level $v$ but paraconsistently with the rest of the inconsistencies of level $\mu$. After doing so jump to another level.

The jumps might be regulated by subdialogues. The choice between procedures 1 and 2 could be part of the agreements either at the very start or, more dynamically, during the dialogue. Here many details have to be fixed, for example: what happens if one constant is ontologically committed but for a given predicate different from those defining the O-inconsistency? We will leave this and other related problems for a future research where we will study the combination of the hierarchy of quantifiers with Newton da Costa's theory of partial truth.

Now although we believe that all this is congenial to the spirit of adaptive logic our approach does not seem to agree exactly with the present adaptive systems developed in Gent. The first difference is that adaptive logic concentrates mainly on how to derive from a given set of formulae. We can emulate this by considering the set of premises as a set of concessions at the very start of the dialogue. Such dialogues are known in the literature as Hypotheses-dialogues (H-dialogues for short). The approach we have presented above seems to agree more with Joke Meheus' system ANA ([17]), as remarked in a personal e-mail by Diderik Batens, for example in that $q$ does but $r$ does not follow from the H-dialogue with initial concessions $\{p, \neg p, p \vee q\}-$ or in the language of formulae of the standard dialogues $((a \vee b) \wedge a \wedge \neg a)) \rightarrow b$ holds but $((a \vee b) \wedge a \wedge \neg a)) \rightarrow r$ does not. In the first case the $\mathrm{O}$-inconsistency produced by the conjunction $a \wedge \neg a$ can be dismissed without hindering the defence $b$. In the second case the round which should be closed with $r$ will remain for ever open. The precise relations between our approach and Joke Meheus' should be studied thoroughly in a future research, but nevertheless we claim that our formulations deserve the adjective adaptive.

We will now describe the tableau system which serves as a basis for checking validity.

\section{$3 \quad$ WINNING STRATEGIES}

As already mentioned, validity is defined in dialogical logic via winning strategies of $\mathrm{P}$, i.e. the thesis $A$ is logically valid iff $\mathrm{P}$ can succeed in defending $A$ against all possible allowed criticism by $\mathrm{O}$. In this case, $\mathrm{P}$ has a winning strategy for $A$. A systematic description of the winning strategies available can be obtained from the following considerations:

If $\mathrm{P}$ is to win against any choice of $\mathrm{O}$, we will have to consider two main different situations, namely the dialogical situations in which $\mathrm{O}$ has stated a (complex) formula and those in which $\mathrm{P}$ has stated a (complex) formula. We call these main situations the $\mathrm{O}$-cases and the $\mathrm{P}$-cases respectively.

In both of these situations another distinction has to be examined:

1. P wins by choosing an attack in the O-cases or a defence in the P-cases, iff he can win at least one of the dialogues he can choose.

2. When $\mathrm{O}$ can choose a defence in the O-cases or an attack in the P-cases, $\mathrm{P}$ can win iff he can win all of the dialogues $\mathrm{O}$ can choose.

The closing rules for dialogical tableaux are the usual ones: a branch is closed iff it contains two copies of the same atomic formula, one stated by $\mathrm{O}$ and the other one by $\mathrm{P}$. A tableau for $(\mathrm{P}) A$ (i.e. starting with $(\mathrm{P}) A)$ is closed iff each branch is closed. This shows that strategy systems for classical and intuitionistic logic are nothing other than the very well known tableau systems for these logics.

For the intuitionistic tableau system, the structural rule about the restriction on defences has to be considered. The idea is quite simple: the tableau system allows all the possible defences (even the atomic ones) to be written down, but as soon as determinate formulae (negations, conditionals, universal quantifiers) of $\mathrm{P}$ are attacked all other $\mathrm{P}$-formulae will be deleted - this is an implementation of the structural rule $\mathrm{R}_{\mathrm{I}} 4$ for intuitionistic logic. Clearly, if an attack on a P-statement causes the deletion of the others, then P can only answer the last attack. Those formulae which compel the rest of P's formulae to be deleted will be indicated with the expression " $\Sigma_{[\mathrm{O}]} \mathrm{O}$ which reads: in the set $\Sigma$ save O's formulae and delete all of P's formulae stated

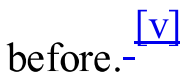




\subsection{Clas sical Table aux}

\begin{tabular}{|c|c|}
\hline (O)-Cases & (P)-Cases \\
\hline$\sum,(\mathrm{O}) A \vee B$ & $\sum,(\mathrm{P}) A \vee B$ \\
\hline$\sum,<(\mathrm{P}) ?>(\mathrm{O}) A \mid \sum,<(\mathrm{P}) ?>(\mathrm{O}) B$ & $\begin{array}{l}\sum,<(\mathrm{O}) ?>(\mathrm{P}) A \\
\sum,<(\mathrm{O}) ?>(\mathrm{P}) B\end{array}$ \\
\hline$\sum,(\mathrm{O}) A \wedge B$ & $\sum,(\mathrm{P}) A \wedge B$ \\
\hline $\begin{array}{l}\sum,<(\mathrm{P}) ? \mathrm{~L}>(\mathrm{O}) A \\
\sum,<(\mathrm{P}) ? \mathrm{R}>(\mathrm{O}) B\end{array}$ & $\sum,<(\mathrm{O}) ? \mathrm{~L}>(\mathrm{P}) A \mid \sum,<(\mathrm{O}) ? \mathrm{R}>(\mathrm{P}) B$ \\
\hline$\sum,(\mathrm{O}) A \rightarrow B$ & $\sum,(\mathrm{P}) A \rightarrow B$ \\
\hline$\sum,(\mathrm{P}) A \ldots \mid<(\mathrm{P}) A>(\mathrm{O}) B$ & $\sum,(\mathrm{O}) A ; \sum,(\mathrm{P}) B$ \\
\hline$\sum,(\mathrm{O}) \neg A$ & $\sum,(\mathrm{P}) \neg A$ \\
\hline$\sum,(\mathrm{P}) A ; \otimes$ & $\sum,(\mathrm{O}) A ; \otimes$ \\
\hline$\sum,(\mathrm{O}) \wedge_{X} A$ & $\sum,(\mathrm{P}) \wedge_{X} A$ \\
\hline$\sum,<(\mathrm{P}) ?_{\tau}>(\mathrm{O}) A_{[\tau / \mathrm{x}]}$ & $\begin{array}{c}\sum,<(\mathrm{O}) ?_{\tau}>(\mathrm{P}) A_{[\tau / \mathrm{x}]} \\
\tau \text { is new }\end{array}$ \\
\hline$\sum,(\mathrm{O}) \vee_{x} A$ & $\sum,(\mathrm{P}) \vee_{x} A$ \\
\hline $\begin{array}{c}\sum,<(\mathrm{P}) ?>(\mathrm{O}) A_{[\tau / \mathrm{x}]} \\
\tau \text { is new }\end{array}$ & $\sum,<(\mathrm{O}) ?>(\mathrm{P}) A_{[\tau / \mathrm{x}]}$ \\
\hline
\end{tabular}

By a dialogically signed formula we mean $(\mathrm{P}) X$ or $(\mathrm{O}) X$ where $X$ is a formula. If $\sum$ is a set of dialogically signed formulae and $X$ is a single dialogically signed formula, we will write $\sum, X$ for $\Sigma \cup\{X\}$. Observe that the formulae below the line always represent pairs of attack and defence moves. In other words, they represent rounds. Note that the expressions between the symbols "<" and " $>$ ", such as $<(\mathrm{P})$ ? $>$ or $<(\mathrm{O})$ ? $>$ are moves more precisely they are attacks - but not statements.

\subsection{Intuitionistic Table aux}

\begin{tabular}{|c|c|}
\hline (O)-Cases & (P)-Cases \\
\hline$\sum,(\mathrm{O}) A \vee B$ & $\sum,(\mathrm{P}) A \vee B$ \\
\hline$\sum,<(\mathrm{P}) ?>(\mathrm{O}) A \mid \sum,<(\mathrm{P}) ?>(\mathrm{O}) B$ & $\begin{array}{l}\sum,<(\mathrm{O}) ?>(\mathrm{P}) A \\
\sum,<(\mathrm{O}) ?>(\mathrm{P}) B\end{array}$ \\
\hline$\sum,(\mathrm{O}) A \wedge B$ & $\sum,(\mathrm{P}) A \wedge B$ \\
\hline $\begin{array}{l}\sum,<(\mathrm{P}) ? \mathrm{~L}>(\mathrm{O}) A \\
\sum,<(\mathrm{P}) ? \mathrm{R}>(\mathrm{O}) B\end{array}$ & $\sum,<(\mathrm{O}) ? \mathrm{~L}>(\mathrm{P}) A \mid \sum,<(\mathrm{O}) ? \mathrm{R}>(\mathrm{P}) B$ \\
\hline$\sum,(\mathrm{O}) A \rightarrow B$ & $\sum,(\mathrm{P}) A \rightarrow B$ \\
\hline$\sum,(\mathrm{P}) A \ldots \mid<(\mathrm{P}) A>(\mathrm{O}) B$ & $\Sigma_{[\mathrm{O}]},(\mathrm{O}) A ;(\mathrm{P}) B$ \\
\hline
\end{tabular}




$$
\begin{gathered}
\sum,(\mathrm{O}) \neg A \\
\sum,(\mathrm{P}) A ; \otimes \\
\sum,(\mathrm{O}) \wedge_{X} A \\
\sum,<(\mathrm{P}) ?_{\tau}>(\mathrm{O}) A_{[\tau / \mathrm{x}]} \\
\sum,(\mathrm{O}) \vee_{x} A \\
\left.---------{ }^{A}\right) A_{[\tau / \mathrm{x}]} \\
\tau,<\text { is new }
\end{gathered}
$$

$$
\begin{gathered}
\sum,(\mathrm{P}) \neg A \\
\sum_{[\mathrm{O}]},(\mathrm{O}) A ; \otimes \\
\sum,(\mathrm{P}) \wedge_{X} A \\
\sum_{[\mathrm{O}],}<(\mathrm{O}) ?_{\tau}>(\mathrm{P}) A_{[\tau / \mathrm{x}]} \\
\tau \text { is new } \\
\sum,(\mathrm{P}) \vee_{x} A \\
\sum,<(\mathrm{O}) ?>(\mathrm{P}) A_{[\tau / \mathrm{x}]}
\end{gathered}
$$

Let us look at two examples, namely one for classical logic and one for intuitionistic logic. We use the tree shape of the tableau made popular by Smullyan ([18]) and omit the expressions between $<$ and $>$ :

Example 6

(P) $\quad \wedge_{x} \neg P_{x} \rightarrow \neg P_{\tau}$

(O) $\wedge_{x} \neg P_{x}$

(P) $\neg P_{\tau}$

(O) $\quad P_{\tau}$

(O) $\neg P_{\tau}$

(P) $\quad P_{\tau}$

The tableau closes.

The following intuitionistic tableau makes use of the deletion rule:

Example 7

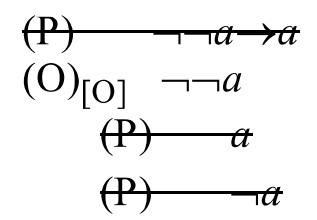

$(\mathrm{O})_{[\mathrm{O}]} a$

The tableau remains open.

\subsection{Winning Strategies for Adaptive Paraconsistency as Tableaux}

In order to introduce a tableau system for dialogical adaptively paraconsistent logic which captures the idea of redundancy described above the rule for closing branches has to be reformulated.

For this aim we will use a notational system which makes use of the device of tagging those formulae which have been used in the sense of our definition of adaptive paraconsistency. More precisely:

Tagging Rule:

1. We will tag any O-atomic formula at least one occurrence of which has been used to close at least one branch (and delete the unused occurrences).

2. We will tag any O-conjunctive formula iff at least one of the conjuncts has been used in at least one branch (and delete the unused conjunct).

3. We will tag any O-complex formula other than a conjunction which has been used in at least one branch.

4. We will tag any P-atomic formula at least one occurrence of which has been used to close at least one branch. 
5. We will tag any P-disjunctive formula iff at least one of the disjuncts has been used in one branch (and delete the unused occurrences).

6. We will tag any P-complex formula other than a disjunction which has been used in at least one branch.

Now the instructions for developing an adaptive tableau:

- If it is possible to close the tableau without producing an inconsistency of the forms described in definition 6 use the standard closing rules. If an inconsistency occurs develop the tableau completely, tag the formulae according to the tagging rule and apply the following adaptive rule.

- A tableau for (P)A (i.e. starting with (P)A) is adaptively closed iff each branch is closed by the standard closing rule and there are no untagged formulae.

\section{Example 8}
i) $\quad(\mathrm{P})(a \wedge \neg a) \rightarrow \neg(a \wedge b)$
ii) $\quad(\mathrm{O})(a \wedge \neg a)$
iii) $\quad(\mathrm{P}) \neg(a \wedge b)$
iv) $\quad(\mathrm{O}) a \wedge b$
v) $\quad<(\mathrm{P})$ ? $\mathrm{L}>(\mathrm{O}) a$
vi) $\quad<(\mathrm{P}) ? \mathrm{R}>(\mathrm{O}) \mathrm{b}$
vii) $\quad<(\mathrm{P})$ ? $\mathrm{R}>(\mathrm{O}) \neg a$

First we use the standard rules and develop the tableau. In doing so we realise that lines $\mathrm{v}$ and vii state an inconsistency. We then develop an adaptive strategy applying the tagging rule. In doing so the Proponent deletes the redundant formula $b$ of the conjunction at move iv and the redundant conjunct $a$ of the conjunction at move ii. The Proponent then uses the resulting atomic formulae to close the branch and the tree:
i) $\quad(\mathrm{P})(a \wedge \neg a) \rightarrow \neg(a \wedge b)$
ii) $\quad(\mathrm{O})(a \wedge \neg a)$
iii) $\quad$ (P) $\neg(a \wedge b)$
iv) $(\mathrm{O}) a \wedge b$
v) $\quad<(\mathrm{P})$ ? $\mathrm{L}>(\mathrm{O}) a$
vi) $\quad<(\mathrm{P})$ ? $\mathrm{R}>(\mathrm{O}) \neg a$
vii) $\quad(\mathrm{P}) a$

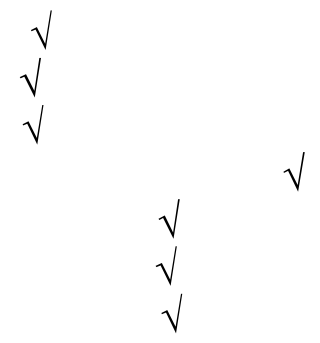

\section{Example 9}
i)
ii)
iii)
iv)
v)
vi)
vii)
ix)

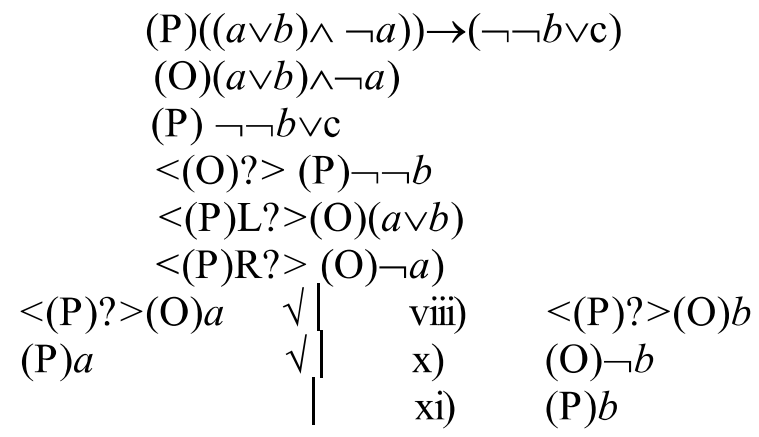
$\sqrt{ }$
$\sqrt{ }$
$\sqrt{ }$
$\sqrt{ }$
$\sqrt{ }$
$\sqrt{ }$
$\sqrt{ }$
$\sqrt{ }$

Each branch is closed. Moreover every formula has been tagged. Thus, everything that should be used has been used and the tableau is adaptively closed.

As far as quantification is concerned we will only suggest how to produce tableau systems with ontologically committed quantifiers and leave the rest of the work to the reader:

Rewrite the rules for the quantifiers in the following way:

\begin{tabular}{c|c}
$(\mathrm{O})$-Cases & $(\mathrm{P})$-Cases \\
\hline &
\end{tabular}




$$
\begin{gathered}
\sum,(\mathrm{O}) \wedge{ }^{\mathrm{i}}{ }_{x} \\
\sum,<(\mathrm{P}){ }_{\tau} *>(\mathrm{O}) A_{[\tau / \mathrm{x}]}
\end{gathered}
$$

$\tau$ has been labelled with an asterisk before

$$
\begin{gathered}
\sum,(\mathrm{O}) \vee^{\mathrm{i}}{ }_{x}^{A} \\
\left.\sum,<(\mathrm{P}) ?>(\mathrm{O}) A_{[\tau} * / \mathrm{x}\right] \\
\tau \text { is new }
\end{gathered}
$$

$$
\begin{aligned}
& \sum,(\mathrm{P}) \wedge^{\mathrm{i}}{ }_{x} A \\
& \Sigma_{[\mathrm{O}]},<(\mathrm{O}) ?_{\tau *>}(\mathrm{P}) A_{[\tau * / \mathrm{x}]} \\
& \tau \text { is new } \\
& \sum,(\mathrm{P}) \vee^{\mathrm{i}}{ }_{x} A \\
& \sum,<(\mathrm{O}) ?>(\mathrm{P}) A_{[\tau * / \mathrm{x}]}
\end{aligned}
$$

DEFINITION 7: A constant $\tau$ is said to be introduced with ontological commitment belonging to the type $\mathrm{i}$ iff it has been labelled with an asterisk by the use of quantifiers of the type $\mathrm{i}$.

\section{$4 \quad$ FINAL REMARKS}

As suggested in the text, several logics which we did not describe may be introduced by changing the notions of redundancy, namely, not allowing any occurrence of an atomic formula to be left unused, not allowing any Oformula to be left unused and not allowing any P-formula to be left unused. This could be combined with the option literal versus complex inconsistencies. Several others result from combining these logics, with the idea of ontological adaptivity.

Neither did we explore how to combine the logical way of eliminating inconsistencies by redundancy with a material strategy for establishing preferences. We will leave this for future research.

\section{APPENDIX: A BRIEF INTRODUCTION TO DIALOGICAL LOGIC}

Dialogical logic, suggested by Paul Lorenzen in 1958 and developed by Kuno Lorenz in several papers from 1961 onwards,,$-\left[\right.$ vi] ${ }^{-}$was introduced as a pragmatic semantics for both classical and intuitionistic logic.

The dialogical approach studies logic as an inherently pragmatic notion using an overtly externalised argumentation formulated as a dialogue between two parties taking up the roles of an Opponent $(\mathrm{O}$ in the following) and a Proponent (P) of the issue at stake, called the principal thesis of the dialogue. $\mathrm{P}$ has to try to defend the thesis against all possible allowed criticism (attacks) by $\mathrm{O}$, thereby being allowed to use statements that $\mathrm{O}$ may have made at the outset of the dialogue. The thesis $A$ is logically valid if and only if $\mathrm{P}$ can succeed in defending $A$ against all possible allowed criticism by $\mathrm{O}$. In the jargon of game theory: $\mathrm{P}$ has a winning strategy for $A$. We will now describe an intuitionistic and a classical dialogical logic.

Suppose the elements and the logical constants of first-order language are given with small italic letters $(a, b, c$, ...) for elementary formulae, capital italic letters for formulae that might be complex $(A, B, C, \ldots)$, capital italic bold letters $(P, Q, R, \ldots)$ for predicators and $\tau_{\mathrm{i}}$ for constants. A dialogue is a sequence of formulae of this firstorder language that are stated by either $\mathrm{P}$ or O.- ${ }^{[\text {vii] }}$-Every move — with the exception of the first move through which the Proponent states the thesis — is an aggressive or a defensive act. In dialogical logic the meaning in use of the logical particles is given by two types of rules which determine their local and their global meaning (particle and structural rules respectively).

The particle rules specify for each particle a pair of moves consisting of an attack and (if possible) the corresponding defence. Each such pair is called a round. A round is opened by an attack and is closed by a defence if one is possible.

\section{PARTICLE RULES}

\begin{tabular}{|l|l|l|}
\hline$\neg, \wedge, \vee, \rightarrow, \wedge, \vee$ & ATTACK & DEFENCE \\
\hline
\end{tabular}




\begin{tabular}{|c|c|c|}
\hline$\neg A$ & $A$ & $\begin{array}{c}\otimes \\
\text { (The symbol ' } \otimes \text { ' } \\
\text { indicates that no } \\
\text { defence, but only } \\
\text { counter-attack is } \\
\text { allowed) }\end{array}$ \\
\hline$A \wedge B$ & $\begin{array}{c}\text { ?L } \\
\text { (The attacker chooses) }\end{array}$ & $\begin{array}{c}A \\
B\end{array}$ \\
\hline$A \vee B$ & $?$ & $\begin{array}{c}A \\
B \\
\text { (The defender chooses) }\end{array}$ \\
\hline$A \rightarrow B$ & $A$ & $B$ \\
\hline$\wedge_{x} A$ & $\begin{array}{c}?_{\tau} \\
\text { (The attacker chooses) }\end{array}$ & $A[\tau / \mathrm{x}]$ \\
\hline$\vee_{x} A$ & $?$ & $\begin{array}{c}A[\tau / \mathrm{x}] \\
\text { (The defender chooses) }\end{array}$ \\
\hline
\end{tabular}

The first column contains the form of the formula in question, the second one possible attacks against this formula, and the last one possible defences against those attacks. (The symbol " $\otimes$ " indicates that no defence is possible.) Note that for example "? L" is a move - more precisely it is an attack - but not a formula. Thus if one partner in the dialogue states a conjunction, the other may initiate the attack by asking either for the lefthand side of the conjunction ("show me that the left-hand side of the conjunction holds", or "?L" for short) or the right-hand side ("show me that the right-hand side of the conjunction holds", or "?R"). If, on the other hand, one partner in the dialogue states a disjunction, the other may initiate the attack by asking to be shown any side of the disjunction ("?").

Next, we fix the way formulae are sequenced to form dialogues with a set of structural rules (orig. Rahmenregeln):

R0 (starting rule):

Moves are alternately uttered by $\mathrm{P}$ and $\mathrm{O}$. The initial formula is uttered by $\mathrm{P}$. It provides the topic of argument. Every move below the initial formula is either an attack or a defence against an earlier move stated by the other player.

\section{$\mathrm{R} 1$ (formal rule for atomic formulae):}

$\mathrm{P}$ may not introduce atomic formulae: any atomic formula must be stated by $\mathrm{O}$ first. Atomic formulae can not be attacked.

\section{$\mathrm{R}_{\mathrm{I}} 2$ (intuitionistic rule):}

In any move, each player may attack a (complex) formula asserted by his partner or he may defend himself against the last not already defended attack. Only the latest open attack may be answered: if it is X's turn at position $v$ and there are two open attacks $\mu, \lambda$ such that $\mu<\lambda<v$, then X may not defend against $\mu$ - $^{-}$

$$
\text { [viii] }
$$

These rules define an intuitionistic logic. To obtain the classical version simply replace $\mathrm{R}_{\mathrm{I}} 2$ by the following rule:

\section{$\mathrm{R}_{\mathrm{C}} 2$ (classical rule):}

In any move, each player may attack a (complex) formula asserted by his partner or he may defend himself against any attack (including those which have already been defended).

Before stating the next rule we need the following definition (the observation about dialogical contexts in the 
following definition is only relevant for relevance logic, modal logic and linear logic with exponentials):

DEFINITION 8: We speak of the strict repetition of an attack iff

a. A move is being attacked although the same move (from the same dialogical context) has already been attacked with the same attack before (notice that ?L and ?R are in this context different attacks).

In the case of moves where a universal quantifier has been attacked with a new constant, the following type of move has to be added to the list of strict repetitions:

b. A universal-quantifier-move is being attacked using a new constant, although the same move (from the same dialogical context) has already been attacked before with a constant which was new at the time of that attack.

DEFINITION 9: We speak of the strict repetition of a defence iff

c. An aggressive move (=attack) 1 which has been already with the defensive move $m$ (=defence) before, is being defended from the challenge at 1 once more with the same defensive formula (notice that the left part and the right part of a disjunction are in this context two different defences)

In the case of moves where an existential quantifier has been defended with a new constant, the following type of move has to be added to the list of strict repetitions:

d. An attack on an existential quantifier is being defended using a new constant although the same quantifier (from the same dialogical context) has already been defended before with a constant which was new at the time of that defence.

\section{R3 (no delaying tactics rule):}

While playing with the classical structural rule $\left(\right.$ see $\left.\mathrm{R}_{\mathrm{C}} 2\right) \mathrm{P}$ may perform a strict repetition of a defence stating $a$ (atomic) twice (or more) if and only if $\mathrm{O}$ has conceded $a$ twice (or more). No other strict repetitions are allowed.

While playing with the intuitionistic structural rule $\mathrm{P}$ may perform a strict repetition of an attack (see $\left.\mathrm{R}_{\mathrm{I}} 2\right)$ if and only if $\mathrm{O}$ has introduced a new atomic formula (see $\mathrm{R} 1$ below) which can now be used by $\mathrm{P}$ (or iff $\mathrm{O}$ has introduced a new dialogical context which is now accessible for $\mathrm{P}$ ).

(Notice that according to the definitions leading to this rule neither the new defence of a existential quantifier nor a new attack on a universal quantifier using a constant different (but not new) from the one used in the first defence (in the first attack) represents a strict repetition)

R4 (winning rule):

$\mathrm{X}$ wins iff it is Y's turn but he cannot move (whether to attack or defend).

As already mentioned, validity is defined in dialogical logic via winning strategies of P:

\section{DEFINITION 10: Validity:}

In a certain dialogical system a formula is said to be valid iff $P$ has a (formal) winning strategy for it, i.e.

$\mathrm{P}$ can in accordance with the appropriate rules succeed in defending $A$ against all possible allowed criticism by $\mathrm{O}^{-}-[\mathrm{ix}]$

It is possible to build tableau systems for winning strategies, which correspond to the well-known semantic tableau methods. We present them in the text.- ${ }^{[\mathrm{x}]}$

Example 10 (either with the classical or the intuitionistic structural rule: it makes no difference):

\begin{tabular}{ll|lll}
\multicolumn{2}{c|}{$\mathrm{O}$} & \multicolumn{3}{c}{$\mathrm{P}$} \\
\hline & & & $((a \rightarrow b) \wedge a) \rightarrow b$ & $(0)$ \\
(1) $\quad(a \rightarrow b) \wedge a$ & 0 & & $b$ & $(8)$ \\
(3) $a \rightarrow b$ & & 1 & $? \mathrm{~L}$ & $(2)$ \\
(5) $\quad a$ & & 1 & $? \mathrm{R}$ & $(4)$ \\
(7) $\quad b$ & & 3 & $a$ & $(6)$ \\
P wins & & & &
\end{tabular}


Example 12 (classical):

\begin{tabular}{|c|c|c|c|c|}
\hline \multicolumn{3}{|c|}{$\mathrm{O}$} & \multicolumn{2}{|l|}{$\mathrm{P}$} \\
\hline & & & $\wedge_{x}\left(P_{x} \vee \neg P_{x}\right)$ & (0) \\
\hline (1) & $?_{\tau}$ & 0 & $P_{\tau} \vee \neg P_{\tau}$ & (2) \\
\hline (3) & $?$ & 2 & $\neg P_{\tau}$ & (4) \\
\hline (5) & $P_{\tau}$ & 4 & $\otimes$ & \\
\hline (3’) & $?$ & 2 & $P_{\tau}^{\otimes}$ & (6) \\
\hline
\end{tabular}

Remarks:

Notation: Moves are labelled in (chronological) order of appearance. They are not listed in the order of utterance, but in such a way that every defence appears on the same level as the corresponding attack. Thus, the order of the moves is labelled by a number between brackets. Numbers without brackets indicate which move is being attacked.

Example 12 shows how the classical structural rule works: the Proponent may, according to the classical structural rules, defend an attack which was not the last one. This allows the Proponent to state $P_{\tau}$ in move (6). For notational reasons we repeated the attack of the Opponent, but actually this move does not take place. That is why, instead of tagging the attack with a new number, we repeated the number of the first attack and added an apostrophe.

The quite simple structure of the dialogue in this and the following examples should make it possible to recognise with the help of only one dialogue whether $\mathrm{P}$ has a winning strategy or not.

The next rule shows how the non-delaying rule works in classical logic:-

Example 13 (classical):

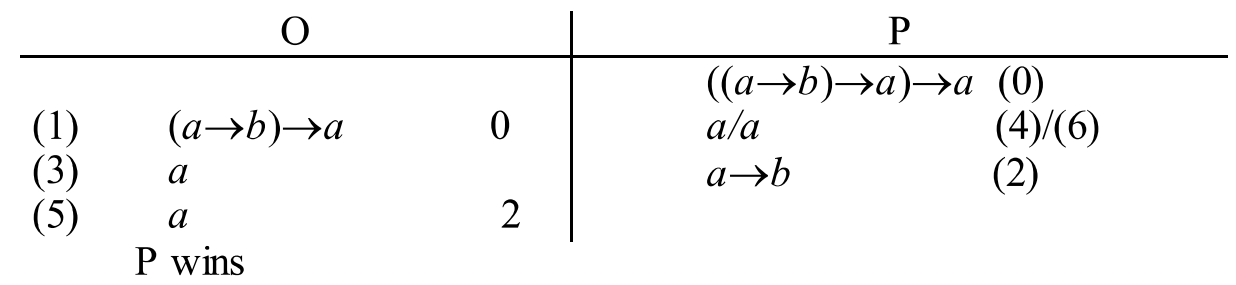

Here, the clever Opponent, who does not give up so easily, after the Proponents s defence 4, attacks move 2 stating $a$ once more. But, the Opponent's twofold use of $a$ allows the Proponent to repeat his defence.

The next two examples deal with dialogues with hypotheses. In these dialogues the Proponent states his thesis under the condition of some hypotheses. Any such hypothesis is to be included as a concession of the Opponent at the very start of the dialogue. The hypotheses will be formulated with schematic letters and the Proponent can at any stage of the dialogue make use of these hypotheses asking first for an adequate instantiation (chosen by the Proponent) of the according schematic letters. ${ }^{[\text {xii] }}$-In the first of the next two examples we show how to obtain tertium non datur from an adequate instantiation of Peirce's Law:

Example 14 (intuitionistic):

\begin{tabular}{ll|lll}
\multicolumn{2}{c|}{$\mathrm{O}$} & \multicolumn{2}{c}{$\mathrm{P}$} \\
\hline $\mathrm{H}:(\mathfrak{R} \rightarrow \mathfrak{I}) \rightarrow \mathfrak{R}) \rightarrow \mathfrak{R}$ & & & \\
$(1)$ & $?$ & & & $(0)$ \\
$(3)$ & $((a \vee \neg a) \rightarrow \neg(a \vee \neg a)) \rightarrow(a \vee \neg a)$ \\
& $\rightarrow(a \vee \neg a)$ & $\mathrm{H}:$ & $?(a \vee \neg a) / \mathfrak{R}, \neg(a \vee \neg a) / \mathfrak{I}$
\end{tabular}


P wins

With move 3, the Proponent asks for an adequate instantiation of the schematic letters. The reader can verify that if at move the Opponent instead of attacking move 4 he defends himself from move 4 he can loose even faster.

Our last example show how to obtain tertium non datur from an adequate instantiation of double negation:

Example 15 (intuitionistic):

\begin{tabular}{|c|c|c|c|c|c|}
\hline & & & & & \\
\hline $\mathrm{H}: \neg$ & $\rightarrow \mathfrak{R}$ & & & $a \vee \neg a$ & (0) \\
\hline (1) & $?$ & 0 & & & \\
\hline (3) & $\neg \neg(a \vee \neg a)$ & & H: & $?(a \vee \neg a) / \mathfrak{R}$ & (2) \\
\hline (5) & $\neg(a \vee \neg a)$ & 4 & 3 & $\neg^{\otimes}(a \vee \neg a)$ & (4) \\
\hline & $\otimes$ & & 5 & $a \vee \neg a$ & (6) \\
\hline (9) & $?$ & 6 & & $\neg a$ & (8) \\
\hline (11) & $a$ & 8 & & $\otimes$ & \\
\hline (13) & $\begin{array}{l}\otimes \\
?\end{array}$ & 10 & 5 & $\begin{array}{l}a \vee \neg a \\
a\end{array}$ & $\begin{array}{l}(10) \\
(12)\end{array}$ \\
\hline
\end{tabular}

P wins

Here the Proponent can repeat an attack on move 5 because the Opponent introduced a new atomic formula at move 11 .

\section{REFERENCES}

[1] Rahman, S. and Carnielli, W.A. The Dialogical Approach to Paraconsistency. In D. Krause, editor, The work of Newton da Costa, special issue of Synthese, 2001. In Press.

[2] Rahman, S. and Roetti, J. A. Dual intuitionistic paraconsistency without ontological commitments. In Proceedings of the International Congress: Analytic Philosophy at the turn of the Millenium in Santiago de Compostela (Spain), 1-4 December 1999, pages 120- 126, 1999.

[3] Rahman, S. Ways of Understanding Hugh MacColl's Concept of Symbolic Existence. Nordic Journal of Philosophical Logic, volume 3 (1-2): 35-58, 1998.

[4] Rahman, S. Argumentieren mit Widersprüchen und Fiktionen. In K. Buchholz, S. Rahman and I. Weber, editors, pages 131-148, Campus, New York-Frankfurt a.M., 1999.

[5] Rahman, S. On Frege's Nightmare. In H. Wansing, editor, Essays on non-classical logic, King's College, London, 2001. In Press.

[6] Van Bendegem, J. P. Paraconsistency and Dialogue Logic. Critical Examination and Further Explorations. In Rahman and Rückert, editors, 2000. In Press.

[7] Batens, D. A survey of inconsistency-adaptive logics. In Press, 2000.

[8] Urchs, M. Recent trends in paraconsistent logics. Konstanz, Technical Report of the DFG-Group Logik in der Philosophie, volume 46, 1999,

[9] Rahman, S. and Rückert, H. Dialogische Logik und Relevanz. FR 5.1 Philosophie, Universität des Saarlandes, volume 27 (Memo), 1998. 
[10] Rahman, S. and Rückert H. Dialogical Modal Logic. Logique et Analyse, 2001, volume March 2001. In Press.

[11] Rahman, S. and Rückert, H. Eine neue dialogische Semantik für die lineare Logik. In C.F. Gethmann, G. Kamp, Neue Entwicklungen der Konstruktiven Logik, 2001. In Press.

[12] Rückert, H. Why Dialogical Logic? In H. Wansing, editor, Essays on non-classical logic, King's College: London, 2001. In Press.

[13] Rückert, H. Dialogue games and connexive logic. In A. Baltag, M. Pauly, editor, Workshop Logic and Games, pages 35-42, ILLC, Amsterdam, 1999.

[14] Da Costa, N. C. A. Paraconsistent Logic. In Stanislaw Jáskowski Memorial Symposium. Paraconsistent Logic, Logical Philosophy, Mathematics \& Informatics at Torún,, pages 29-35, 1998.

[15] Da Costa, N. C. A; Bueno, O. and French, S. The Logic of Pragmatic Truth. Journal of Philosophical Logic. volume 26: 603-620, 1998.

[16] Rahman, S., Rückert, H. and Fischmann, M.. On Dialogues and Ontology. The dialogical approach to free logic. Logique et Analyse, 1997, volume 160,327-374.

[17] Meheus, J. Rich paraconsistent logics: the three-valued logic AN and the adaptive logic ANA that is based on it. In print.

[18] Smullyan, R.. First-Order Logic. Heidelberg, Springer, 1968.

[19] Lorenzen, P. and Lorenz, K. Dialogische Logik. Wissenschaftliche Buchgesellschaft, Darmstadt, 1978.

[20] Rahman, S. Über Dialoge, protologische Kategorien und andere Seltenheiten. Peter Lang, Frankfurt a. M.-NewYork-Paris, 1993.

[21] Krabbe, E. C. W. Formal Systems of Dialogue Rules. Synthese, volume 63(3): 295-328, 1985.

[22] Rahman, S. and Rückert, H. Die pragmatischen Sinn- und Geltungskriterien der Dialogischen Logik beim Beweis des Adjunktionssatzes. Philosophia Scientiae, volume 3(3): 145-170, 1999.

[23] Felscher, W. Dialogues, strategies and intuitionistic provability. Annals of Pure and Applied Logic, volume 28: 217-254, 1985.

[24] Rahman, S. and Rückert, H., editors. New Perspectives in Dialogical Logic. Special issue of Synthese with contributions of P. Blackburn, D. Gabbay and J. Woods, J. Hintikka, E. Krabbe, K. Lorenz, U. Nortmann, H. Prakken, S. Rahman and H. Rückert, G. Sandu, J. P. Van Bendegem, G. Vreeswijk, volume April 2001, 2001. In Press.

\footnotetext{
* We would like to thank Diderik Batens (Ghent), Gerhard Heinzmann (Nancy), for helpful philosophical and his torical comments on the subject of this paper, João Marcos (Unicamp) and Helge Rückert (Saarbrücken / Leiden) for critical comments on an earlier version, and Graham Priest (Queensland) for sending us unpublished material on the topic. The first author would like to thank the CNPq for financial support of position as a visiting-researcher at the University of Campinas where he had the opportunity to discuss with high-level researchers about the issues developed in this paper.

[i] -For a short introduction to standard dialogical logic see the appendix.

[ii] -See details on how to build the tableau systems in Rahman and Carnielli ([1]) and Rahman ([3] and [5]).

[iii] -João Marcos (Unicamp) observed that the classical literal system corresponds to Sette's P1.

[iv] -A systematic formulation of subdialogues for relevant aims can be found in Rahman and Rückert ([10] and [11]) and
} 
Rückert ([12] and [13]).

[vi]

-Cf. [19]. Further work has been done for example by Rahman ([20]).

[vii]

-Sometimes we use $\mathbf{X}$ and $\mathbf{Y}$ to denote $\mathbf{P}$ and $\mathbf{O}$ with $\mathbf{X} \neq \mathbf{Y}$.

[viii]

-Notice that this does not mean that the last open attack was the last move.

[ix]

-See consistency and completeness theorems in Krabbe ([21]) and Rahman ([20]).

$[\mathrm{x}]$

-See more details on how to build tableau systems from dialogues in Rahman ([20]), Rahman and Rückert ([22]).

Alternative systems with the corresponding proofs have been given by Felscher ([23]) and by Krabbe ([21]).

[xi]

-João Marcos (Campinas), used this example in a seminar offered by Rahman at the Unicamp in order to show that earlier versions of the non-delaying rule should be reformulated with more precision.

[xii]

-Actually in the case of dialogues with hypotheses we need to extend the non-delaying rule in such a way that unnecessary repetitions of instantiations should be avoided. This extension is not difficult if we think that an aggressive instantiation-move works in a similar way as an attack on a universal quantifier: the Proponent may repeat instantiationattacks until all propositional variables occurring in the thesis have been used for an instantiation. We leave the details for the reader. 